\title{
PERCEPÇÃO DE INDÍGENAS MUNDURUKU E EQUIPE MULTIDISCIPLINAR DE SAÚDE INDİGENA SOBRE RESOLUTIVIDADE NA ATENÇÃO À SAÚDE
}

\author{
Bahiyyeh Ahmadpour Furtado ${ }^{1}$, David Lopes Neto ${ }^{2}$, Daniel Scopel ${ }^{3}$, Raquel Paiva Dias-Scopel ${ }^{4}$
}

Objetivo: compreender as percepções de indigenas da etnia Munduruku e de profissionais da Equipe Multidisciplinar de Saúde Indígena sobre a resolutividade no Pólo-base Kwatá, Terra Indígena Kwatá Laranjal, localizada no município de Borba, estado do Amazonas, Brasil. Metodologia: os dados foram coletados por meio de observação participante, entrevistas e análise documental. O trabalho de campo foi realizado entre agosto e outubro de 2015. Resultados: os achados mostraram que usuários e profissionais perceberam deficiências na resolução dos problemas de saúde e identificaram obstáculos para a efetivação da Política Nacional de Atenção à Saúde dos Povos Indígenas. Conclusão: a resolutividade foi compreendida como a junção de diferentes práticas tradicionais somadas às práticas integrativas e complementares, com garantia da preservação da cultura indigena no processo de cuidar.

Descritores: Resolução de Problemas; Serviços de Saúde; Saúde de Populações Indígenas.

\section{PERCEPTION OF MUNDURUKU INDIGENOUS AND MULTIDISCIPLINARY TEAM OF INDIGENOUS HEALTH ABOUT RESOLUTENESS IN HEALTH CARE}

Objective: This study aimed to understand the perceptions of Munduruku indigenous and multidisciplinary team of indigenous health on the resoluteness in Kwatá, Indigenous Land Kwatá Laranjal, located in the municipality of Borba, Amazonas state, Brazil. Data were collected through participant observation, interviews and document analysis. The field work was conducted between august and october 2015. The results showed that users and professionals perceive shortcomings in resoluteness health problems and identified obstacles to the realization of brazilian policy health care of indigenous peoples.

Descriptors: Problem Solving; Health Service; Health of Indigenous Peoples.

\section{PERCEPCIÓN DE INDÍGENA MUNDURUKUY EQUIPO MULTIDISCIPLINARIO DE SALUD INDİGENA SOBRE LA RESOLUCIÓN EN LAATENCIÓN EN SALUD: OBSTÁCULOS Y DESAFÍOS}

Objetivo: Este estudio tuvo como objetivo comprender las percepciones de equipo multidisciplinario indígenas Munduruku étnica y profesional de la salud indígena en la solución de problemas de Kwatá, tierra indígena Kwata-Laranjal, ubicado en el municipio de Borba, estado de Amazonas, Brasil. Los datos fueron recolectados a través de la observación participante, entrevistas y análisis de documentos. El trabajo de campo se realizó entre agosto y octubre de 2015. Los resultados mostraron que los usuarios y los profesionales perciben deficiencias en la solución de los problemas de salud y los obstáculos identificados para la realización de la Política Nacional para el Cuidado de la Salud de los Pueblos Indígenas.

Descriptores: Solución de problemas; Servicios de Salud; Salud de Poblaciones Indígenas.

${ }^{1}$ Enfermeira. Mestre em Enfermagem. Docente da Faculdade de Medicina da Universidade Federal do Amazonas - UFAM.

Enfermeiro. Doutor em Enfermagem. Docente da Escola de Enfermagem de Manaus da Universidade Federal do Amazonas - UFAM. E-mail: davidnetto@uol.com.br ${ }^{3}$ Doutor em Antropologia Social. Pesquisador do Instituto Leônidas e Maria Deane - ILMD/FIOCRUZ.

${ }^{4}$ Doutora em Antropologia Social. Pesquisadora da Fundação Oswaldo Cruz/Instituto Leônidas e Maria Deane/ILMD/Manaus. 


\section{INTRODUÇÃO}

Desde 1957, a temática da saúde indígena vem sendo debatida nos campos da antropologia, da saúde, da educação. A partir da criação no Sistema Único de Saúde (SUS), do Subsistema de Atenção à Saúde Indigena (SASI) (Lei no 9.836, de 1999), o Ministério da Saúde implementou uma rede de serviços nas terras indígenas de forma a superar as deficiências de cobertura, acesso e aceitabilidade do SUS por meio de convênios com organizações não governamentais e municipios $^{(1,2)}$.

O SASI organiza-se a partir da atenção primária com niveis crescentes de complexidade técnica e a partir de áreas geográficas delimitadas pelos Distritos Sanitários Especiais Indigenas (DSEI). A atenção primária é a porta de entrada e preferencial da rede de atenção, acolhendo aos usuários e promovendo a vinculação e corresponsabilização às necessidades de saúde ${ }^{(3)}$. Cada DSEI organiza-se a partir de um conjunto de pólos-base e respectivas Equipes Multidisciplinares de Saúde Indígena (EMSI). As EMSI são compostas, minimante, por médicos, enfermeiros, dentistas, técnicos de enfermagem e agentes indígenas de saúde e saneamento.

A resolutividade é um dos princípios do SUS e pode ser definida como a capacidade de cada instância organizativa resolver os problemas relativos ao nível de competência estabelecido hierarquicamente ${ }^{(4-6)}$. Como resposta satisfatória do serviço de saúde às necessidades do usuário, a resolutividade avalia os resultados a partir da prestação de serviços da demanda interna e por meio dos encaminhamentos a serviços especializados ${ }^{(7)}$.

Por esse motivo, vários autores ${ }^{(8-10)}$ têm utilizado a perspectiva dos usuários e profissionais de saúde para avaliar resolutividade em saúde, considerando-se que a percepção do usuário sobre a qualidade dos serviços expressa o grau de satisfação com o acesso, articulação e continuidade das ações entre os diferentes níveis de hierarquia organizacional, podendo servir como parâmetro para identificar obstáculos à resolutividade e estabelecer desafios.

Este artigo tem como objetivo compreender as percepções de indígenas da etnia Munduruku e de profissionais da Equipe Multidisciplinar de Saúde Indígena sobre a resolutividade no Pólo-base Kwatá.

\section{METODOLOGIA}

Desenho metodológico etnográfico, de interpretaçãocompreensiva, com combinação mista de métodos, abordagens e técnicas qualitativas, do observação participante e entrevista semiestruturada.

O contexto local da pesquisa foi a aldeia Kwatá, situada às margens do rio Canumã, na Terra Indígena (TI) Kwatá-
Laranjal, município de Borba, estado do Amazonas. A língua nativa dos Munduruku é do tronco Tupi(11), com predominância do idioma português.

O trabalho de campo foi realizado no período entre agosto e outubro de 2015, totalizando 20 dias. Na primeira viagem, acompanhou-se o atendimento da EMSI em oito aldeias. $\mathrm{Na}$ segunda viagem, realizou-se observação participante no polo base Kwatá e na CASAI de Nova Olinda do Norte. As observações foram registradas em diários de campo. Foram realizadas cinco entrevistas com profissionais de saúde (uma enfermeira, três técnicas de enfermagem e um médico) e 14 entrevistas com indigenas (10 Agentes Indígenas de Saúde (AIS) e quatro lideranças - pajés e parteira indígena).

As informações coletadas foram organizadas por meio da técnica de análise de conteúdo, com leituras flutuantes do corpus, seleção das unidades de análise temática (resolutividade) e categorização não-apriorística para classificação dos elementos das respostas dos participantes do estudo em duas subcategorias: obstáculos relacionados a recursos materiais, financeiros e humanos.

Pesquisa aprovada pelo Comitê de Ética em Pesquisa da Universidade Federal do Amazonas e pela Comissão Nacional de Ética em Pesquisa, CAAE: 38527014.7.0000.5020.

\section{RESULTADOS}

A resolutividade no subsistema de atenção à saúde indígena, narrada pelo Indígena 1, advém, primeiramente, das práticas integrativas complementares e tradicionais indigenas quando esse diz que ainda preservamos resolver nossos problemas de saúde através de ervas medicinais e através dos cuidados dos pajés.

Os profissionais de saúde destacaram como obstáculos à resolutividade na atenção à saúde indígena a escassez de equipamentos e de materiais, as frequentes interrupções no fornecimento de energia elétrica, o que impossibilita o estoque de produtos imunobiológicos e medicamentos refrigerados.

"... nós temos os medicamentos, porém não conseguimos medir a glicemia das pessoas, pois não temos o aparelho,..." (médico). "Alguns materiais faltam aqui no polo, materiais para sutura, por exemplo, precisamos de mais para fazer curativos e são poucos pela quantidade de demanda que aparecem por aqui no polo (Téc. de Enfermagem 1).

Para os AIS, a falta de recurso financeiro para compra de combustivel é um problema, haja vista que combustivel é para a remoção até o polo, e eu recebo $10 \mathrm{~L}$ por mês que não é suficiente (AIS 5). O combustível é pouco porque eu pego 30L de gasolina por mês, tem vezes que a escola empresta para nós, e quando eu pego a cota eu devolvo o mais rápido possivel (AIS 6).

A escassez de profissionais de saúde na EMSI é fator 
condicionante da baixa resolutividade em área indígena. A dificuldade em contratar e manter profissionais de saúde em área gera deficiências na qualidade da atenção diferenciada. Técnicas de enfermagem narraram sentir sobrecarga de trabalho e necessidade de distribuir as responsabilidades do cotidiano de trabalho para desempenhar suas funções com eficiência. Suas percepções evidenciaram que alguns veem o trabalho da enfermagem de maneira positiva, mas tem outros que criticam, mas na maioria acham bom ter o polo aqui com as técnicas e enfermeira, mas temos poucas pessoas, só uma enfermeira e quatro técnicas. Se tivesse mais membros na equipe poderia fazer mais visitas nas aldeias (Téc. de Enfermagem 2).

$\mathrm{Na}$ percepção dos AIS, a rotatividade dos profissionais é ruim para nós, pois nós criamos um vínculo com a profissional, e depois ela vai embora. Nos primeiros meses a enfermeira começa a trabalhar aqui com empolgação, mas depois diminui a velocidade e produtividade. Normalmente as enfermeiras que trabalham aqui elas vão embora para fazer cursos na cidade e em outros lugares e acabam não voltando mais (Indigena 1).

\section{DISCUSSÃO}

A disponibilidade dos profissionais de saúde em área indígena não restringe o itinerário terapêutico dos indígenas quanto ao modelo tecnoassistencial de saúde, por não ser, o Pólo-base, o único local procurado pelos indígenas para atendimento, mesmo representando o modelo biomédico hegemônico ${ }^{(3,11,12,13)}$.

Pesquisa etnográfica sobre uso e disponibilidade de medicamentos entre os Guarani mostrou que a atenção primária àquelas populações indígenas era marcada pelo "assistencialismo hipermedicalizante de caráter curativo"(14), estando a disponibilidade de medicamentos associada à presença das equipes de saúde na aldeia, sendo o médico o "principal prescritor".

Estudo entre os indígenas Baniwa ${ }^{(15)}$ verificou que a disponibilidade e circulação de medicamentos estavam relacionadas ao poder e prestígio local das lideranças indígenas e, pesquisa sobre o uso de medicamentos em rituais xamânicos entre os Aguaruna verificou que a apropriação de medicamentos refletia a assimetria das relações coloniais e o poder simbólico dos medicamentos associado à presença do poder estatal na Amazônia ${ }^{(16)}$. Para os indígenas Munduruku, a falta de medicamentos está relacionada a precariedade dos serviços de saúde. No caso dos AIS Munduruku, seus discursos sobre falta de medicamentos relacionavam-se com a perda de atribuições e prerrogativas de prescrição de medicamentos, configurando o desempoderamento do fazer médico em paralelo à (des)estruturação das EMSI.

Para os AIS e EMSI, a falta de combustivel (gasolina) é obstáculo à resolutividade dos problemas de saúde pela essencialidade na remoção de pacientes graves, gestantes com intercorrências e deslocamento dos profissionais para visitas às aldeias.

As dificuldades de transporte, seja no âmbito da atenção primária no interior das terras indígenas, seja na remoção de pacientes até os centros urbanos para atenção na rede do SUS, também emergiram como fatores de impacto negativo na resolutividade dos serviços de saúde, conforme relatado por outros estudos ${ }^{(17,18)}$

Notadamente, a escassez de profissionais de saúde impacta negativamente em todas as atividades planejadas e programadas e a rotatividade desses dificulta a aquisição de habilidades e competências para o diálogo intercultural ${ }^{(19)}$. A alta rotatividade de profissionais da EMSI, a sobrecarga de trabalho, as dificuldades de comunicação, a baixa reposição de materiais e de equipamentos emergiram neste estudo e emergem em outras pesquisas como fatores que impactam na qualidade da atenção básica em diferentes contextos indígenas no Brasil(3,20,21).

Para Cardoso ${ }^{(22)}$, os modelos assistencial e organizacional do SASI não estão consolidados, o que contribui para a baixa resolutividade do subsistema. Para a autora, a atenção básica ofertada pelos DSEI está marcada por "carências de profissionais habilitados para atender a essa população, alta rotatividade dos profissionais em área, falta de recursos de infraestrutura e equipamentos para determinados procedimentos e ações operados pelos DSEI, assim como a relativa falta de integração e um sistema de comunicação eficaz com aquele de referência do SUS".

Investigação sobre o papel de profissionais de saúde na atenção aos Munduruku do Pará mostrou haver uma polarização nas atitudes de profissionais de saúde, motivados por sentimentos de benevolência e pelo racionalismo burocrático. Essas motivações implicariam a continuidade das formas coloniais de administração, etnocentrismo e submissão dos indígenas ao poder do Estado, contradizendo os princípios da PNASI ${ }^{(23)}$.

As articulações e confrontos entre os saberes indigenas e o processo de medicalização apontaram as características estruturais da expansão do modelo médico hegemônico na atenção à saúde indígena Munduruku, expressado pela junção dos saberes tradicionais e biomédico. Por outro lado, os profissionais de saúde ainda tendem a negar e ignorar práticas advindas dos sabres tradicionais e as perspectivas e expectativas indígenas ${ }^{(11)}$. $O$ modelo organizacional de saúde indígena preconiza a necessidade de recursos humanos indígenas e não indígenas em áreas indígenas, com competência cultural, epidemiológica para o cuidar culturalmente congruente com as diferentes realidades das aldeias $^{(24)}$. 


\section{CONCLUSÃO}

O estudo analisou a percepção de indigenas Munduruku e profissionais de saúde que atuam em área indígena sobre a resolutividade na atenção à saúde. Os interlocutores indígenas assinalaram a importância das práticas tradicionais de saúde, assim como das práticas inerentes do modelo biomédico.

A resolutividade foi compreendida como a junção de diferentes práticas tradicionais somadas às práticas integrativas e complementares, com garantia da preservação da cultura indígena no processo de cuidar.
Os obstáculos à resolutividade em saúde são recorrentes na aldeia Kwatá e estão relacionados à escassez e turnover de profissionais de saúde, inefetividade da gestão de recursos materiais, tanto permanente quanto de consumo, pela consequente escassez de recursos financeiros.

E, por fim, como em meio aos obstáculos narrados pelos profissionais de saúde e indígenas, como desafios para superá-los faz-se necessário um olhar transcultural sobre a Política Nacional de Atenção à Saúde Indígena para a reorganização do SASI, especialmente no que se refere à estrutura de recursos humanos, materiais e financeiros.

\section{REFERÊNCIAS}

1. Garnelo L. Política de Saúde Indígena no Brasil: notas sobre as tendências atuais do processo de implantação do subsistema de atenção à saúde. In: Garnelo L, Pontes AL, organizadores. Saude Indigena: Uma Introdução ao Tema [Internet]. Brasilia: MEC-SECADI; 2012 [citado 17 de abril de 2013]. p. 19-58. Recuperado de: http://www.trilhasdeconhecimentos.etc.br/livros/ arquivos/ColET15_Vias05WEB.pdf

2. Diehl EE, Langdon EJ. Transformações na Atenção à Saúde Indigena: Tensões e Negociações em um Contexto Indigena Brasileiro. Univ Humanistica. 2015;80(80)

3. Ministério da Saúde. Política nacional de atenção à saúde dos povos indigenas. 2. ed. Brasilia: FUNASA; 2002.

4. Ministério da Saúde. Abc do SUS: Doutrinas e Principios [Internet]. Brasilia, DF; 1990. Recuperado de: http://www.pbh.gov.br/smsa/ bibliografia/abc_do_sus_doutrinas_e_principios.pdf

5. Turrini RNT, Lebrão ML, Cesar CLG. Resolutividade dos serviços de saúde por inquérito domiciliar: percepção do usuário Case-resolving capacity of health care services according to a household survey: users perceptions. Cad Saúde Pública. 2008;24(3):663-74.

6. Rosa RB, Pelegrini AH, Lima MAD da S. Resolutividade da assistência e satisfação de usuários da estratégia saúde da familia. Rev Gaúcha Enferm. 32(2), 2011:345-351.

7. Costa JP Jorge MSB, Vasconcelos MGF, Paula ML, Bezerra IC. Resolubilidade do cuidado na atenção primária: articulação multiprofissional e rede de serviços. Saúde Debate, 2014:38(103):733-43.

8. Ministério da Saúde. Secretaria de Gestão Estratégica e Participativa. Política Nacional de Gestão Estratégica e Participativa no SUS ParticipaSUS [Internet]. Brasilia, DF: Editora MS; 2009. Recuperado de: http://bvsms.saude.gov.br/bvs/publicacoes/politica_estrategica_ participasus_2ed.pdf

9. Ribeiro JP. Acesso e resolutividade: satisfação dos usuários de serviços de proteção às vítimas de violência intrafamiliar [Dissertação de Mestrado]. Rio Grande: Programa de Pós-graduação em enfermagem, Universidade Federal do Rio Grande; 2011.

10. Silva RMM da, Viera CS, Toso BRG de O, Neves ET, Rodrigues RM. Resolutividade na atenção à saúde da criança: percepção de pais e cuidadores. Acta Paul Enferm. 2013;26(4):382-8.

11. Dias-Scopel RP. A cosmopolítica da gestação, parto e pós-parto: práticas de autoatenção e processo de medicalização entre os Indios Munduruku [Tese de doutorado]. Florianópolis: Programa de Pósgraduação em Antropologia Social, Universidade Federal de Santa Catarina. 2014. 211 p.

12. Menéndez EL. Sujeitos, Saberes e Estruturas: uma introdução ao enfoque relacional no estudo da saúde coletiva. São Paulo SP Brasil: Hucitec: 2009.437 p.

13. Langdon EJ. Os diálogos da antropologia com a saúde: contribuições para as politicas públicas. Rev Ciênc Saúde Coletiva. 2014;19(4).

14. Diehl EE, Grassi F. Use of medicines in a Guarani Indian village on the coast of Santa Catarina State, Brazil. Cad Saúde Pública. 2010:26(8):154960.

15. Garnelo L. Poder, hierarquia e reciprocidade: saúde e harmonia entre os Baniwa do Alto Rio Negro. Rio de Janeiro: Editora Fiocruz; 2003.

16. Greene S. The Shaman's Needle: Development, Shamanic Agency, and Intermedicality in Aguaruna Lands, Peru. Am Ethnol. 1998:25(4):634-58.

17. Ghiggi Junior A. Uma abordagem relacional da atenção à saúde a partir da terra indigena Xapecó.[Tese de Doutorado]. Florianópolis: Programa de Pós-Graduação em Antropologia Social, Universidade Federal de Santa Catarina; 2015. $378 p$.

18. Dias-Scopel RP. O agente indigena de saúde Xokleng: por um mediador entre a comunidade indígena e o serviço de atenção diferenciada à saúde-uma abordagem da antropologia da saúde [Dissertação de Mestrado]. Florianópolis: Programa Pós-graduação em Antropologia Social, Universidade Federal de Santa Catarina: 2005.

19. Langdon EJ. Medicina Tradicional: reflexões antropológicas sobre atenção diferenciada. In: Haverroth M, organizador. Etnobiologia e Saúde dos Povos Indigenas. Recife: Nupeea; 2013. (Estudos Avançados).

20. Dias-Scopel RP, Langdon EJ, Scopel D. Expectativas emergentes: a inserção do agente indigena de saúde Xokleng na equipe multidisciplinar de saúde indigena. Tellus. 2007:13:51-73.

21. Gusman CR, Viana AP de AL, Miranda MAB, Pedrosa MV. Villela WV. Inclusão de parteiras tradicionais no Sistema Único de Saúde no Brasil: reflexão sobre desafios. Rev Panam Salud Pública. maio de 2015;37(45):365-70.

22. Cardoso MD. Políticas de saúde indigena no Brasil: do modelo assistencial à representação política. In: Langdon EJ, Cardoso MP. organizadores. Saúde indigena : politicas comparadas na América Latina. Florianópolis: Editora UFSC; 2015. (Coleção Brasil Plural).

23. Silva CD da. De Improvisos e Cuidados: a saúde indigena e o campo da enfermagem. In: Teixeira CC, Garnelo L, organizadores. Saúde dos povos indigenas. Editora Fiocruz; 2014

24. Lopes Neto D, Furtado BA. Saúde Indigena. In: Associação Brasileira de Enfermagem. Kalinowski CE, Crozeta K, Costa MFBNA. PROENF Programa de Atualização em Enfermagem. Atenção Primária e Saúde da familia: Ciclo 4. Porto Alegre: Artmed Panamericana, 2016. p.117-50. 